Pacific Journal of Mathematics

ON THE CONVERGENCE OF CLOSED AND COMPACT SETS 


\section{ON THE CONVERGENCE OF CLOSED AND COMPACT SETS}

\section{Eva LOWEN-COLEBUNDERS}

For a topological Hausdorff space $X$ we study the hyperspaces $\mathscr{P}(X), 2^{X}$ and $\mathcal{C}(X)$ of all closed subsets, all non-empty closed subsets and all non-empty compact subsets endowed with the convergence of sets. In this paper we shall work with the filter description of this convergence, as defined by Choquet [2], which however is equivalent to the topological convergence of nets of sets as defined by Frolik and Mrówka. We shall study the relation between properties of $X$ and properties of the spaces $\mathscr{P}(X), 2^{X}$ and $\varrho(X)$ such as compactness, local compactness, regularity and the topological and pretopological character.

1. Introduction. The aim of this paper is to study properties of the convergence of closed or compact sets of a Hausdorff topological space. The class $\mathscr{P}(X)$ of all closed subsets of a Hausdorff topological space $X$, will be endowed with the natural pseudotopological structure of closed convergence. On $2^{X}$, the collection of non-empty closed subsets of $X$ and on $\mathcal{C}(X)$, the collection of non-empty compact subsets of $X$, we consider the induced pseudotopological structures. The main purpose is to investigate under what conditions on $X$, the spaces $\mathscr{P}(X), 2^{X}$ and $\mathcal{C}(X)$ are compact, locally compact, regular, topological or pretopological. The pseudotopological structure of closed convergence has been defined by Choquet in [2, p. 87] using the supremum (Sup) and the infimum (inf) of filters on $\mathscr{P}(X)$. If $\chi$ is a filter on $\mathscr{P}(X)$ and $p \in X$ we have $p \in \operatorname{Sup} \chi$ if and only if for each neighborhood $V$ of $p$ and for each $Q \in \chi$ there exists an $A \in \mathbb{Q}$ such that $A \cap V \neq \varnothing$. Analogously $p \in \operatorname{Inf} \chi$ if and only if for each neighborhood $V$ of $p$ there exists an $Q \in \chi$ such that for each $A \in \mathbb{Q}$, $A \cap V \neq \varnothing$. A filter $\chi$ is said to converge to some $A \in \mathcal{P}(X)$ if and only if $\operatorname{Sup} \chi=\operatorname{Inf} \chi=A$.

This structure has been studied in this form or in an equivalent form for net convergence in [2], [4], [5], [10], [11]. It generalizes the notion of closed convergence of sequences introduced by Hausdorff [6].

For all notational conventions and definitions on convergence spaces we refer to [1], [2], [3], [8]. We recall some definitions and notations that will be used frequently.

Let $X$ be a topological space. A subset $A$ of $X$ is relatively compact if and only if every ultrafilter containing $A$ has a limit in $X$. A subset of $X$ 
which has a compact closure is relatively compact. The converse holds for regular spaces but not in general [1, p. 98]. A topological space is said to be locally relatively compact if and only if every point has a relatively compact neighborhood.

In a pseudotopological space $X$ the closure of a set $A$ is denoted by $\bar{A}$. For $x \in X$ we have $x \in \bar{A}$ if and only if there exists a filter $\mathscr{F}$ containing $A$ and converging to $x . A$ is said to be dense if $\bar{A}=X$, closed if $\bar{A}=A$ and open if $A^{c}$ is closed. Then also $A$ is open if and only if every filter converging to some point of $A$ contains $A$.

The adherence of a filter $\mathscr{F}$ is denoted by $\alpha \mathscr{F}$ and it is the set of limits of the filters finer than $\mathcal{F}$.

For each $x \in X$ the neighborhoodfilter $\Re(x)$ is the intersection of all filters converging to $x$. We have $\mathscr{B}(x)=\{A \mid x \notin \overline{X \backslash A}\}$. $\mathscr{B}(x)$ converges to $x$ for every $x \in X$ if and only if $X$ is pretopological. If in addition $\mathscr{B}(x)$ has an open base for every $x \in X$ then $X$ is topological. A pseudotopological space is Hausdorff if every filter has at most one limit and regular if $\overline{\mathscr{F}}$ converges to $x$ whenever $\mathscr{F}$ converges to $x$, where $\overline{\mathscr{F}}=[\{\vec{F} \mid F \in \mathscr{F}\}]$. The space is compact if every ultrafilter has a limit and it is locally compact if every convergent filter contains a compact set. In the sequel $X$ will be a Hausdorff topological space containing at least two points.

2. The space $\mathscr{P}(X)$. For a filter $\chi$ on $\mathscr{P}(X)$ let $\operatorname{Sup} \chi$ and $\operatorname{Inf} \chi$ be defined as in the previous section. Another characterization of Sup $\chi$ will be useful. If $\mathscr{Q} \in \chi$ then we define $E_{\mathscr{Q}}=\cup\{A \mid A \in \mathbb{Q}\}$. In [2, p. 61] it is shown that $\operatorname{Sup} \chi=\bigcap\left\{\bar{E}_{\mathscr{Q}} \mid \mathbb{Q} \in \chi\right\}$. If $\chi \neq \dot{\varnothing}$ then $\left\{E_{\mathbb{Q}} \mid \mathbb{Q} \in \chi\right\}$ is a filterbase on $X$. Let $\mathscr{F}(\chi)$ be the filter generated. Then we have $\operatorname{Sup} \chi=$ $\alpha_{X} \mathscr{F}(\chi)$. For any filter $\chi$ on $\mathscr{P}(X)$ we have $\operatorname{Inf} \chi \subset \operatorname{Sup} \chi$. For ultrafilters $\chi$ we have $\operatorname{Inf} \chi=\operatorname{Sup} \chi[2$, p. 62]. Now let $\mathscr{P}(X)$ be endowed with the Choquet structure.

Identifying $x$ and $\{x\}, X$ can be considered a subspace of $\mathscr{P}(X)$. The space $\mathscr{P}(X)$ is known to be a compact Hausdorff pseudotopology which is topological if and only if $X$ is locally compact [4], [11].

THEOREM (2.1). The following properties are equivalent:

(1) $X$ is locally compact,

(2) $\mathscr{P}(X)$ is topological,

(3) $\mathscr{P}(X)$ is regular,

(4) $\mathscr{P}(X)$ is pretopological.

Proof. (1) $\Rightarrow$ (2) follows from the results of [4] and [11].

$(2) \Rightarrow(3)$ follows from the compactness of $\mathscr{P}(X)$. 
(3) $\Rightarrow$ (4) follows from the theorem that every regular compact Hausdorff pseudotopology is topological. [12, p. 572]

$(4) \Rightarrow(1)$. Suppose $\mathscr{P}(X)$ is pretopological. It is sufficient to prove that $X$ is locally relatively compact and regular. If $X$ is not locally relatively compact then there is an $x \in X$ such that the neighborhoodfilter $\mathcal{V}(x)$ of $x$ does not contain a relatively compact set. For each $V \in \mathcal{V}(x)$ we choose an ultrafilter $\mathcal{U}_{V}$ containing $V$ and having no limit in $X$. Let $\mathscr{}$ be an ultrafilter finer than $\mathscr{V}(x) \vee \cap\left\{\mathcal{Q}_{V} \mid V \in \mathcal{V}(x)\right\}$. Now let's consider $X$ as a subspace of $\mathscr{P}(X)$. Then $\mathscr{W}$ generates a filter [W] on $\mathscr{P}(X)$ converging to $\{x\}$ and for any $V \in \mathcal{V}(x), \mathcal{Q}_{V}$ generates a filter $\left[\mathcal{Q}_{V}\right]$ converging to $\varnothing$. Since $[\mathscr{Q}] \supset \cap\left\{\left[\mathcal{Q}_{V}\right] \mid V \in \mathscr{V}(x)\right\}$ it follows that the neighborhoodfilter of $\varnothing$ in $\mathscr{P}(X)$ will not converge to $\varnothing$. Hence $\mathscr{P}(X)$ is not pretopological.

If $X$ is not regular then let $E$ be a non-empty closed subset of $X$ such that $E \neq \cap\{\bar{V} \mid V \in \mathscr{V}(E)\}$ where $\mathscr{V}(E)$ is the neighborhoodfilter of $E$ in $X$. Let $f_{E}: X \rightarrow \mathscr{P}(X)$ be the function mapping $x$ on $\{x\} \cup E$. For any $x \in E$ the filter $f_{E}(\mathscr{V}(x))$ converges to $E$ in $\mathcal{P}(X)$ because $\mathscr{F}\left(f_{E}(\mathscr{V}(x))\right)=$ $\mathcal{V}(x) \cap[E]$ and thus $\operatorname{Sup} f_{E}(\mathscr{V}(x))=E$. Clearly we also have $E \subset$ Inf $f_{E}(\mathscr{V}(x))$. We have $f_{E}(\mathscr{V}(E))=\bigcap_{x \in E} f_{E}(\mathcal{V}(x))$ and $\mathscr{F}\left(f_{E}(\mathcal{V}(E))\right)=$ $\mathcal{V}(E) \cap[E]$. Hence $\operatorname{Sup} f_{E}(\mathcal{V}(E)) \neq E$ and so $f_{E}(\mathcal{V}(E))$ does not converge to $E$. It follows that $\mathcal{P}(X)$ is not pretopological.

3. The space $2^{X}$. Now we consider the collection $2^{X}$ of all nonempty closed subsets of $X$ with the convergence induced by $\mathcal{P}(X) .2^{X}$ is an open subspace of $\mathscr{P}(X)$ and clearly $2^{X}$ is closed in $\mathscr{P}(X)$ if and only if $X$ is compact. So $2^{X}$ is compact if and only if $X$ is compact as was stated by Choquet [2, p. 88].

If $X$ is locally compact then $\mathscr{P}(X)$ is a compact topological space and so the open subspace $2^{X}$ is a locally compact topological space [2, p. 88], $\left[10\right.$, p. 241]. Mrówka has shown that if $X$ is regular and $2^{X}$ is topological then $X$ is locally compact [10, p. 242]. We show the stronger result that even without the regularity assumption the local compactness of $X$ follows from the pretopological character of $2^{X}$.

THEOREM (3.1). Each of the following properties of $2^{X}$ are equivalent to the local compactness of $X$ :

(1) $2^{X}$ is pretopological,

(2) $2^{X}$ is topological,

(3) $2^{X}$ is locally compact,

(4) $2^{X}$ is regular. 
Proof. That the local compactness of $X$ implies (1), (2), (3) and (4), was explained in the previous remarks. We now prove the other implications.

(1) $\Rightarrow X$ is locally compact: Suppose $2^{X}$ is pretopological. We shall prove that $\mathscr{P}(X)$ is also pretopological. Then $X$ is locally compact as was shown in the previous theorem.

Let $A \in \mathscr{P}(X)$ and let $\left(\chi_{j}\right)_{j \in J}$ be a family of filters on $\mathscr{P}(X)$ converging to $A$. If $A \neq \varnothing$ then for each $j \in J$ we have $\operatorname{Inf} \chi_{j} \neq \varnothing$ and hence $2^{X} \in \chi_{j}$. So $2^{X} \in \bigcap_{j \in J} \chi$, and therefore $\bigcap_{j \in J}\left(\chi_{j} / 2^{X}\right)$ converges to $A$ in $2^{X}$. It follows that $\bigcap_{j \in J} \chi_{j}$ converges to $A$ in $\mathcal{P}(X)$. If $A=\varnothing$ then $\operatorname{Sup} \chi_{J}=\varnothing$ for every $j \in J$. We may assume that $\chi_{J} \neq \dot{\varnothing}$ for each $j \in J$. Let $\mathscr{F}_{j}=\mathscr{F}\left(\chi_{J}\right)$, let $\chi=\bigcap_{J \in J} \chi_{j}$ and $\mathscr{F}(\chi)=\mathscr{F}$. Then $\mathscr{F}=\bigcap_{j \in J} \mathscr{F}_{j}$.

Now take any non-empty closed subset $E$ of $X$ and consider the associated map $f_{E}: X \rightarrow 2^{X}$ as in Theorem 2.1. Then the filters $f_{E}\left(\mathscr{F}_{j}\right)$ all converge to $E$ in $2^{X}$. Since $2^{X}$ is pretopological we have that $f_{E}(\mathscr{F})=$ $\bigcap_{, \in J} f_{E}\left(\mathscr{F}_{j}\right)$ converges to $E$. But then we have $\operatorname{Sup} f_{E}(\mathscr{F})=\alpha_{X} \mathscr{F} \cup E=E$ so that $\alpha_{X} \mathscr{F} \subset E$. Since this is true for any choice of a non-empty closed set $E$ we have $\alpha_{X} \mathscr{F}=\varnothing$ and $\chi$ converges to $\varnothing$ in $\mathscr{P}(X)$. It follows that $\mathscr{P}(X)$ is pretopological.

(2) $\Rightarrow X$ is locally compact follows at once from the previous result.

(3) $\Rightarrow X$ is locally compact: Suppose $2^{X}$ is locally compact. We first show that $X$ always is a closed subset of $2^{X}$ (cfr. [9, Prop. 1.8]). Let $\chi$ be a filter on $2^{X}$ converging to some $E \in 2^{X}$ and containing $X$. If $E$ contains more than one element choose $x_{1}$ and $x_{2}$ in $E, x_{1} \neq x_{2}$. Let $V_{1}$ and $V_{2}$ be disjoint neighborhoods of $x_{1}$ and $x_{2}$. Let $\mathbb{Q}_{1} \in \chi\left(\right.$ and $\left.\mathscr{Q}_{2} \in \chi\right)$ have the property that for any $A \in \mathbb{Q}_{1}\left(A \in \mathbb{Q}_{2}\right), V_{1} \cap A \neq \varnothing\left(V_{2} \cap A \neq \varnothing\right)$. Then $\mathbb{Q}_{1} \cap \mathbb{Q}_{2} \cap X \neq \varnothing$ which is impossible. Now since $X$ is closed in $2^{X}$, using the closed hereditarity of local compactness [8] it follows that $X$ is locally compact.

(4) $\Rightarrow X$ is locally compact: Suppose that $2^{X}$ is regular. Then since $X$ is a subspace it is also regular. Therefore it is sufficient to show that $X$ is locally relatively compact. Suppose on the contrary that $X$ is not locally relatively compact. As in Theorem 2.1 let $x$ be a point of $X$ such that $\mathscr{V}(x)$ does not contain a relatively compact set. As in Theorem 2.1 we construct the family $\left(\mathcal{Q}_{V}\right)_{V \in \mathcal{V}(x)}$ and $\mathcal{} \supset \mathcal{V}(x) \vee \cap\left\{\mathcal{Q}_{V} \mid V \in \mathcal{V}(x)\right\}$. We take $E$ closed and non-empty and not containing $x$ and we consider the associated map $f_{E}$. For any $V \in \mathcal{V}(x)$ the filter $f_{E}\left(\mathcal{Q}_{V}\right)$ converges to $E$ and $f_{E}(\mathscr{W})$ converges to $E \cup\{x\}$ in $2^{X}$. Since $f_{E}($ W $) \supset \bigcap_{V \in \mathscr{W}(x)} f_{E}\left(\mho_{V}\right)$ we have $\dot{E} \supset \overline{f_{E}(\mathscr{W})}$ and hence $\overline{f_{E}(\mathscr{W})}$ does not converge to $E \cup\{x\}$. This is a contradiction. 
4. The space $\mathcal{C}(X)$. Now we consider the collection $\mathcal{C}(X)$ of all non-empty compact subsets of $X$ with the convergence induced by $2^{X}$. $\mathcal{C}(X)$ is a dense subspace of $2^{X}$ as follows from the fact that the collection of non-empty finite subsets of $X$ is already dense in $2^{X}$ [9, Prop. 1.8].

THEOREM 4.1. The following properties are equivalent:

(1) $X$ is compact,

(2) $\mathcal{C}(X)$ is compact,

(3) $\mathcal{C}(X)$ is locally compact,

(4) $\mathcal{C}(X)$ is open in $2^{X}$.

Proof. (1) $\Rightarrow(2)$ : If $X$ is compact then $\mathcal{C}(X)=2^{X}$.

$(2) \Rightarrow(3)$ is trivial.

(3) $\Rightarrow(4)$ : If $\mathcal{C}(X)$ is locally compact then $X$ is locally compact since it is a closed subspace (cfr. Theorem 3.1).

It follows that $2^{X}$ and $\mathcal{C}(X)$ are topologies and that $\mathcal{C}(X)$ is locally closed in $2^{X}\left[1\right.$, p. 103]. But $\mathcal{C}(X)$ is dense in $2^{X}$ so $\mathcal{C}(X)$ must be open in $2^{X}$.

(4) $\Rightarrow(1)$ : Suppose $X$ is not compact and let $थ$ be an ultrafilter on $X$ with an empty adherence. Let $K$ be a compact non-empty subset of $X$ and put $\mathscr{F}=\overline{\mathscr{Q}} \cap[K]$. Then we have $\alpha_{X} \mathscr{F}=K$. For $F \in \mathscr{F}$ let $\mathbb{Q}_{F}=\left\{E \in 2^{X} \mid\right.$ $E \subset F\}$. Then $\left\{\mathscr{Q}_{F} \mid F \in \mathscr{F}\right\}$ is a filterbase on $2^{X}$. Let $\Gamma(\mathscr{F})$ be the filter generated. Then $\Gamma(\mathscr{F}) \vee \mathcal{C}(X)^{c}$ exists because for each $F \in \mathscr{F}$ we have an $U \in \mathcal{U}$ with $\bar{U} \cup K \subset F$ and so $\bar{U} \in \mathbb{Q}_{F} \cap \mathcal{C}(X)^{c}$. We first show that there exists an ultrafilter $\chi$ on $2^{X}$ finer than $\Gamma(\mathscr{F}) \vee \mathcal{C}(X)^{c}$ and such that $\mathscr{F}(\chi) \subset \mathscr{F}$. Suppose on the contrary that for any ultrafilter $\chi \supset \Gamma(\mathscr{F}) \vee$ $\mathcal{C}(X)^{c}$ we have $\mathscr{F}(\chi) \nsubseteq \mathscr{F}$. Then for any ultrafilter $\chi \supset \Gamma(\mathscr{F}) \vee \mathcal{C}(X)^{c}$, choose $\mathcal{Q}(\chi) \in \chi$ such that $E_{\mathscr{Q}(\chi)} \notin \mathscr{F}$. Then we can find a finite number of these ultrafilters, say $\chi_{1}, \ldots, \chi_{n}$ such that $\cup_{i=1}^{n} \mathscr{Q}\left(\chi_{i}\right) \in \Gamma(\mathscr{F}) \vee \mathcal{C}(X)^{c}$. Otherwise the collection $\Gamma(\mathscr{F}) \vee \mathcal{C}(X)^{c}$ together with all the $\mathscr{Q}(\chi)^{c}$ would generate a filter which is impossible. Now let $U \in \mathcal{Q}$ be such that for $F=\bar{U} \cup K$ we have $\mathbb{Q}_{F} \cap \mathcal{C}(X)^{c} \subset \cup_{i=1}^{n} \mathbb{Q}\left(\chi_{i}\right)$. Since $E_{\mathscr{Q}\left(x_{i}\right)} \not \supset F$ for each $i \in\{1, \ldots, n\}$, choose $\left\{x_{1}, \ldots, x_{n}\right\}, x_{i} \in F$ and such that $x_{i} \notin E_{\mathscr{Q}\left(x_{i}\right)}$ for each $i \in\{1, \ldots, n\}$. Since $F$ is closed we have $F \in \mathbb{Q}_{F} \cap \mathcal{C}(X)^{c}$ so there exists a $j \in\{1, \ldots, n\}$ with $F \in \mathbb{Q}\left(\chi_{j}\right)$. Since $x_{j} \in F$ we would have $x_{j} \in E_{\mathfrak{Q}\left(\chi_{j}\right)}$ which is a contradiction. Now let $\chi$ be an ultrafilter finer than $\Gamma(\mathscr{F}) \vee \mathcal{C}(X)^{c}$ and such that $\mathscr{F}(\chi) \subset \mathscr{F}$. Then clearly we have $\mathscr{F}(\chi)=\mathscr{F}$ and hence $\chi$ converges to $K$ in $2^{X}$. Since $e(X)^{c} \in \chi$ we have that $\mathcal{C}(X)$ is not open. 
THEOREM 4.2. $\mathcal{C}(X)$ is pretopological if and only if $X$ is locally relatively compact.

Proof. Suppose $\mathcal{C}(X)$ is pretopological. We again use the technique of proof of the case $A=\varnothing$ in (1) Theorem (3.1). We choose $E$ to be a non-empty compact subset of $X$ in order to show that the neighborhoodfilter of $\varnothing$ in $\mathscr{P}(X)$ converges to $\varnothing$. This implies that $X$ is locally relatively compact as was shown in the first part of the proof of Theorem 2.1.

For the converse suppose $X$ is locally relatively compact. Let $\left(\chi_{j}\right)_{j \in J}$ be a family of filters on $\mathcal{C}(X)$ converging to some $E \in \mathcal{C}(X)$. Let $\chi=\bigcap_{j \in J} \chi_{j}$. We show that $\chi$ converges to $E$ in $\mathcal{C}(X)$. We have $\operatorname{Inf} \chi=$ $\cap_{J \in J} \operatorname{Inf} \chi_{j}[2$, p. 62] and so $\operatorname{Inf} \chi=E$.

We prove that $\sup \chi \subset E$. Take $\chi \in \operatorname{Sup} \chi$ and let $\mathscr{Q}$ be an ultrafilter, थつ $\mathcal{V}(x) \vee \mathscr{F}(\chi)$. Then we have $X \mid E \notin \overline{\mathcal{Q}}$. Because if we would have $X \mid E \in \overline{\mathcal{Q}}$ then we could find a $U \in \mathcal{Q}$ such that $\bar{U} \subset X \mid E$. Take a relatively compact set $R$ contained in $U$ and belonging to $\mathscr{U}$ then $\bar{R} \subset$ $X \mid E$. For $j \in J$ let $\mathscr{F}\left(\chi_{j}\right)=\bigcap_{i \in I_{j}} \mathcal{Q}_{i}^{j}$ for some index set $I_{j}$ and some family of ultrafilters $\left(\mathcal{Q}_{i}^{j}\right)_{i \in I_{j}}$. Then we have

$$
\mathscr{F}(\chi)=\bigcap_{j \in J} \mathscr{F}\left(\chi_{j}\right)=\bigcap_{j \in J} \bigcap_{i \in I_{j}} \mathscr{Q}_{i}^{j} \subset \mathscr{Q} .
$$

Using [7, Prop. 2] it follows that there exist $j_{0} \in J$ and $i_{0} \in I_{j_{0}}$ such that $R \in \mathcal{Q}_{i_{0}}^{j_{0}}$. Hence $\mathcal{Q}_{i_{0}}^{j_{0}}$ converges to some $y \in X \mid E$. But on the other hand $y \in \operatorname{Sup} \mathscr{F}\left(\chi_{j}\right)=E$ which is a contradiction. Now since $X \mid E \notin \overline{\mathcal{Q}}$ we have that the collection $\{\bar{U} \cap E \mid U \in \mathcal{Q}\}$ has the finite intersection property. Since $E$ is compact we have $\cap_{U \in \mathcal{U}} \bar{U} \cap E=E \cap \alpha_{X}$ $थ=$ $E \cap\{x\} \neq \varnothing$ and thus $x \in E$.

THEOREM 4.3. The following properties are equivalent:

(1) $X$ is locally compact,

(2) $\mathcal{C}(X)$ is regular,

(3) $\mathcal{C}(X)$ is topological.

Proof. (1) $\Leftrightarrow(2)$ : If $X$ is locally compact then $2^{X}$ is regular (3.1) and so is its subspace $\mathcal{C}(X)$. Conversely if $\mathcal{C}(X)$ is regular then its subspace $X$ is regular and it only remains to be shown that $X$ is locally relatively compact. The proof is completely analogous to the proof of (4) in Theorem (3.1) now choosing for $E$ a non-empty compact subset of $X$ not containing $x$. 
$(1) \Leftrightarrow(3)$ : If $X$ is locally compact then we already know that $2^{X}$ is topological. Then so is its subspace $\mathcal{C}(X)$.

For the converse suppose $C(X)$ is topological. From the previous theorem we know that $X$ is locally relatively compact. Since $X$ is Hausdorff and contains more than one point, for any $x \in X$ we can find a relatively compact neighborhood $R$ of $x$, such that $\bar{R} \neq X$. Lets consider the collections

$$
\mathcal{C}_{R}=\{K \mid K \in \mathcal{C}(X), K \cap R \neq \varnothing\}
$$

and

$$
e_{\bar{R}}=\{K \mid K \in \mathcal{C}(X), K \cap \bar{R} \neq \varnothing\} .
$$

We shall show that $\bigodot_{\bar{R}}$ is closed. For this purpose we first prove that $e_{\bar{R}}=\bar{\bigodot}_{R}$. If $K \in \mathcal{C}_{\bar{R}}$ then let $y \in K \cap \bar{R}$ and let $\mathscr{Q}$ be an ultrafilter converging to $y$ and containing $R$. Consider the function $f_{K}: X \rightarrow \mathcal{C}(X)$ mapping $z$ to $\{z\} \cup K$. Then $f_{K}(\mathscr{Q})$ converges to $K$ in $\mathcal{C}(X)$. Since $f_{K}(R) \subset \mathcal{C}_{R}$ we have $K \in \bar{\bigodot}_{R}$. For the other inclusion let $K \in \overline{\mathcal{C}}_{R}$ and let $\chi$ be a filter on $\mathcal{C}(X)$ converging to $K$ and containing $\bigodot_{R}$. Then $\mathscr{F}(\chi) \vee R$ exists since for $Q \in \chi$ we have $Q \cap \mathcal{C}_{R} \neq \varnothing$ and hence $E_{\mathscr{Q}} \cap R \neq \varnothing$. Let $\mathcal{Q}$ be an ultrafilter finer than $\mathscr{F}(\chi) \vee R$. Since $R$ is relatively compact $\mathcal{Q}$ converges to some $y \in \bar{R}$. But $\mathscr{U} \supset \mathcal{F}(\chi)$ so we have $y \in K$ and therefore $K \in \mathcal{C}_{\bar{R}}$. Since $\mathcal{C}(X)$ is supposed to be topological its closure operator is idempotent and so $\bar{C}_{R}$ is closed. Hence we also have that $\bigodot_{\bar{R}}$ is closed. From this we show that $\bar{R}$ is compact. Suppose $\bar{R}$ is not compact. Let $Q$ be an ultrafilter containing $\bar{R}$ and such that $\alpha_{X}$ ข $=\varnothing$. Since $\bar{R} \neq X$ we can choose a compact set $K$ such that $K \cap \bar{R}=\varnothing$. Consider the associated function $f_{K}$ and the ultrafilter $f_{K}(\mathscr{W})$ on $\mathcal{C}(X)$. Then $f_{K}(\mathcal{W})$ converges to $K$ in $\mathcal{C}(X)$ and $\mathcal{C}_{\bar{R}} \in f_{K}(W)$. Hence we have $K \in \overline{\mathcal{C}}_{\bar{R}}=\mathcal{C}_{\bar{R}}$. This is a contradiction. Now since $R$ is compact we have that $x$ has a compact neighborhood which proves that $X$ is locally compact.

\section{REFERENCES}

[1] N. Bourbaki, Topologie Générale, 4th ed. Eléments de Mathématique, Fas II, Chap 1 (Hermann, Paris 1965).

[2] G. Choquet, Convergences, Ann. Univ. Grenoble Sect. Sci. Math. Phys., 23 (1948), 57-112.

[3] H. Fischer, Limesraüme, Math. Ann., 137 (1959), 269-303.

[4] Z. Frolik, Concerning topological convergence of sets, Czechoslovak Math. J., 10 (1960), 168-180.

[5] W. Gähler, Beiträge zur Theorie der Limesraüme, Theory of sets and topology, Berlin, (1972), 161-197.

[6] F. Hausdorff, Mengenlehre, (Berlin-Leipzig, 1927). 
[7] D. Kent, Decisive convergence spaces, Math. Ann., 184 (1970), 215-223.

[8] D. Kent and G. Richardson, Locally compact convergence spaces, Michigan Math. J. 22 (1975), 353-360.

[9] E. Lowen-Colebunders, The Choquet hyperspace structure for convergence spaces, Math. Nachr., 95 (1980), 17-26.

[10] S. Mrówka, On the convergence of nets of sets, Fund. Math., 45 (1958), 237-246.

[11] Some comments on the space of subsets, Lecture Notes in Math., 171 (1970), 59-63.

[12] G. Richardson and D. Kent, Regular compactifications of convergence spaces, Proc. Amer. Math. Soc., 11(1972), 571-573.

Received October 4, 1979 and in revised form July 26, 1982.

VRIJE UNIVERSITEIT BRUSSEL

PleinlaAn 2

B-1050 BRUSSELS, BELGIUM 


\section{PACIFIC JOURNAL OF MATHEMATICS EDITORS}

Donald BABBITT (Managing Editor)

University of California

Los Angeles, CA 90024

Hugo Rossi

University of Utah

Salt Lake City, UT 84112

C. C. Moore and Arthur Ogus

University of California

Berkeley, CA 94720
J. DugundiI

Department of Mathematics

University of Southern California

Los Angeles, CA 90089-1113

R. Finn and H. SAMELSON

Stanford University

Stanford, CA 94305

ASSOCIATE EDITORS
R. ARENS
E. F. BECKENBACH
B. H. NeUmanN
F. WOLF
K. YosHIDA (1906-1982)

\section{SUPPORTING INSTITUTIONS}

UNIVERSITY OF ARIZONA

UNIVERSITY OF BRITISH COLUMBIA

CALIFORNIA INSTITUTE OF TECHNOLOGY

UNIVERSITY OF CALIFORNIA

MONTANA STATE UNIVERSITY

UNIVERSITY OF NEVADA, RENO

NEW MEXICO STATE UNIVERSITY

OREGON STATE UNIVERSITY
UNIVERSITY OF OREGON

UNIVERSITY OF SOUTHERN CALIFORNIA

STANFORD UNIVERSITY

UNIVERSITY OF HAWAII

UNIVERSITY OF TOKYO

UNIVERSITY OF UTAH

WASHINGTON STATE UNIVERSITY

UNIVERSITY OF WASHINGTON 


\section{Pacific Journal of Mathematics}

\section{Vol. 108, No. $1 \quad$ March, 1983}

Waleed A. Al-Salam and A. Verma, $q$-Konhauser polynomials $\ldots \ldots \ldots \ldots 1$

Alfred David Andrew, The Banach space JT is primary $\ldots \ldots \ldots \ldots \ldots . . .6$

Thomas E. Bengtson, Bessel functions on $P_{n} \ldots \ldots \ldots \ldots \ldots \ldots$

Joaquim Bruna Floris and Francesc Tugores, Free interpolation for

holomorphic functions regular to the boundary $\ldots \ldots \ldots \ldots \ldots \ldots \ldots \ldots$

Peter Dierolf and Susanne Dierolf, Topological properties of the dual pair

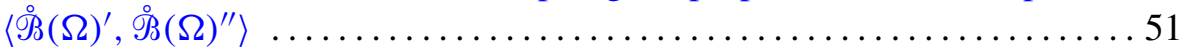

Gerald Arthur Edgar, An ordering for the Banach spaces $\ldots \ldots \ldots \ldots \ldots 83$

Basil Gordon, A proof of the Bender-Knuth conjecture . . . . . . . . . . . . . 99

Harold T. Hodes, A minimal upper bound on a sequence of Turing degrees

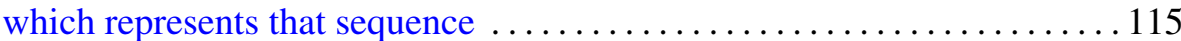

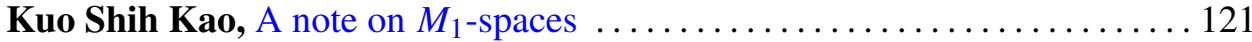

Frank Kost, Topological extensions of product spaces ................ 129

Eva Lowen-Colebunders, On the convergence of closed and compact

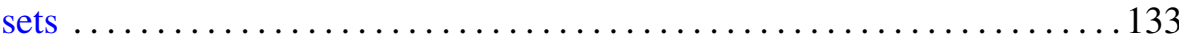

Doron Lubinsky, Divergence of complex rational approximations . . . . . . 141

Warren May and Elias Hanna Toubassi, Endomorphisms of rank one

mixed modules over discrete valuation rings $\ldots \ldots \ldots \ldots \ldots \ldots \ldots \ldots \ldots$

Richard Patrick Morton, The quadratic number fields with cyclic

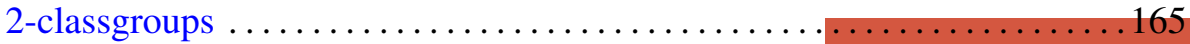

Roderic Murufas, Rank of positive matrix measures . . . . . . . . . . . . 177

Helga Schirmer, Fixed point sets of homotopies . . . . . . . . . . . . . 191

E. Taflin, Analytic linearization of the Korteweg-de Vries equation ........ 203

James Thomas Vance, Jr., $L^{p}$-boundedness of the multiple Hilbert

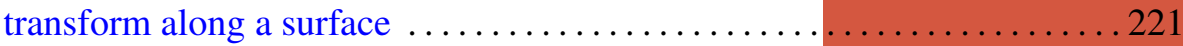

Hiroshi Yamaguchi, A property of some Fourier-Stieltjes transforms . . . . . 243 